\title{
Deep brain stimulation and glioma
}

\author{
Maria Feychting ${ }^{1}$ (D)
}

Received: 2 March 2016 / Accepted: 9 March 2016 / Published online: 23 March 2016

(C) Springer-Verlag Wien 2016

Human exposures to electromagnetic fields are ubiquitous in modern society, and the number of new applications is constantly increasing. Introduction of new technologies, such as mobile telephony, has raised public concern about potential health effects from exposures to electromagnetic fields at levels far below current guidelines $[1,8]$. This has led to a substantial increase in research efforts within this area, despite the absence of known biological mechanisms by which disease would occur at such low exposure levels. The energy generated by electromagnetic fields at such low exposure levels is too weak to break chemical bonds, and well-known interaction mechanisms of electromagnetic fields with the human body induce health effects at considerably higher exposure levels than those encountered in everyday life. Reference levels of international guidelines for workers and the general population are set to protect against such health effects [3, 4]. Nevertheless, research focused on exposure levels below current guidelines is continuing, to ensure that no hitherto unknown biological mechanisms exist.

Along these lines, a case report is published in this issue, where a high-grade glioma was observed in a patient who had previously been treated with deep brain stimulation (DBS) [7]. The authors speculate that electric fields associated with the treatment could have caused

Maria Feychting

maria.feychting@ki.se

1 Institute of Environmental Medicine, Karolinska Institutet, Box 210, SE-171 77 Stockholm, Sweden the tumour. The authors also mention a case report describing the development of glioblastoma in two patients who had cochlear implants, where it was suggested that the tumours might be attributed to the electromagnetic fields from the implants [5]. Both of these are interesting observations, although alternative explanations for the occurrence of the tumours in these patients may be at least as, or even more, plausible. It should be noted that the electromagnetic field exposures from these two applications are very different, with different properties; DBS is used to achieve stimulation of specific areas of the brain through electric impulses. Thus, with DBS, a biological effect is the goal of the treatment, whereas cochlear implants use radiofrequency fields to transfer sound over a very short distance, at field levels that, according to current knowledge, are too low to cause biological effects outside the normal physiological variation. To properly test the hypothesis that the electromagnetic fields from DBS or cochlear implants causes glioma, systematic epidemiological studies need to be conducted, where sizeable cohorts of patients who have undergone DBS or had cochlear implants are observed with regard to the occurrence of glioma over a sufficiently long time period, and where the observed glioma incidence is compared to the incidence in the general population of comparable age and sex distribution. As confounding from the surgical procedures necessary to apply the devices cannot be excluded, additional support from epidemiological studies of other electromagnetic field exposure sources would also be needed, as well as support from experimental studies. Currently, the evidence in support of the hypothesis that radiofrequency fields from mobile phone use increase the risk of glioma is very 
limited [1, 8]. Increased risks have been reported by a few case-control studies that have assessed exposure through retrospectively collected self-reported information about mobile phone use, which is prone to recall bias, and reported risk increases have often been implausible in the light of the stable glioma incidence trends worldwide since the introduction of handheld mobile phones $[2,6]$. In addition, cohort studies that have collected exposure information independently of the disease have found no evidence of increased glioma risks [1, 8]. Nevertheless, the potential effect on glioma risk from the electromagnetic field exposure sources discussed in this issue is worthwhile investigating in systematic epidemiological studies.

\section{Compliance with ethical standards}

Conflicts of interest Maria Feychting is co-investigator of the COSMOS cohort study, funded by the Swedish Research Council, the Swedish Council for Working Life and Social Research, AFA Insurance, and VINNOVA (The Swedish Governmental Agency for Innovation Systems). VINNOVA received funds for this purpose from TeliaSonera, EricssonAB, and Telenor. The provision of funds to the COSMOS study investigators via VINNOVA is governed by agreements that guarantees COSMOS' complete scientific independence.

Maria Feychting is vice chairman of the International Commission on Non-Ionizing Radiation Protection, an independent body setting guidelines for non-ionizing radiation protection. She serves as advisor to a number of national and international public advisory and research steering groups concerning the potential health effects of exposure to non-ionizing radiation.

\section{References}

1. AGNIR (2012) Health effects from radiofrequency electromagnetic fields. Report from the Independent Advisory Group on NonIonising Radiation. Documents of the Health Protection Agency, Radiation, Chemical and Environmental Hazards. RCE 20, Health Protection Agency, London

2. Deltour I, Auvinen A, Feychting M, Johansen C, Klaeboe L, Sankila R, Schuz J (2012) Mobile phone use and incidence of glioma in the Nordic countries 1979-2008: consistency check. Epidemiology 23: 301-307. doi:10.1097/EDE.0b013e3182448295

3. ICNIRP (1998) Guidelines for limiting exposure to time-varying electric, magnetic, and electromagnetic fields (up to $300 \mathrm{GHz}$ ). International Commission on Non-Ionizing Radiation Protection. Health Phys 74:494-522

4. ICNIRP (2010) Guidelines for limiting exposure to time-varying electric and magnetic fields (1 Hz to $100 \mathrm{kHz}$ ). Health Phys 99: 818-836. doi:10.1097/HP.0b013e3181f06c86

5. Kalakoti P, Murray R, Pettersson-Segerlind J, Smeds H, Nanda A (2016) Cochlear implants in the etio-pathogenesis of glioblastomaan interesting observation or an independent finding? Acta Neurochir (Wein). doi:10.1007/s00701-016-2718-3

6. Little MP, Rajaraman P, Curtis RE, Devesa SS, Inskip PD, Check DP, Linet MS (2012) Mobile phone use and glioma risk: comparison of epidemiological study results with incidence trends in the United States. BMJ 344:e1147. doi:10.1136/bmj.e1147

7. Mindermann T, Mendelowitsch A (2016) Deep brain stimulation and development of a high-grade glioma - incidental or causal association? Acta Neurochir (Wein). This issue

8. SCENIHR (2015) Scientific Committee on emerging and newly identified health risks: potential health effects of exposure to electromagnetic fields (EMF). http://ec.europa.eu/health/scientific committees/emerging/docs/scenihr_o_041.pdf. Accessed 15 Aug 2015 